\title{
Moros, Accius et Silena, Orlando : trois aspects de la folie dans le théâtre pré-shakespearien
}

Francis Guinle

\section{(2) OpenEdition}

Journals

\section{Édition électronique}

URL : http://journals.openedition.org/shakespeare/1313

DOI : 10.4000/shakespeare.1313

ISSN : 2271-6424

Éditeur

Société Française Shakespeare

\section{Édition imprimée}

Date de publication : 1 novembre 1989

Pagination : 73-84

Référence électronique

Francis Guinle, « Moros, Accius et Silena, Orlando : trois aspects de la folie dans le théâtre préshakespearien », Actes des congrès de la Société française Shakespeare [En ligne], 7| 1989, mis en ligne le 01 janvier 2007, consulté le 01 mai 2019. URL : http://journals.openedition.org/shakespeare/1313 ; DOI : 10.4000/shakespeare.1313 


\title{
Société Française Shakespeare
}

\author{
Actes des Congrès \\ 1985 - 1986 - 1987
}

\section{LA FOLIE}

\section{SHAKESPEARE ET LES ARTS \\ LE TRAGIQUE}




\section{MOROS, ACCIUS ET SILENA, ORLANDO : TROIS ASPECTS DE LA FOLIE DANS LE THEATRE PRE-SHAKESPEARIEN}

Francis GUINLE

Les personnages cités dans le titre de cette communication se trouvent dans trois pièces pré-shakespeariennes : Moros dans The Longer Thou Livest, The More Fool Thou Art (1559, W. Wager), Accius et Silena dans Mother Bombie (1589, John Lyly), et Orlando dans Orlando Furioso (1591, Robert Greene).

Le théâtre pré-shakespearien étant, dans son ensemble, peu connu, et assez difficile d'accès (malgré un effort ces dernières années, l'édition reste fragmentaire), nous avons tenu compte, dans notre démarche que peu de gens seront très familiers avec ces pièces. Cette démarche paraîtra nécessairement linéaire, mais peut-être suscitera-t-elle un intérêt, incitant chacun à faire plus ample connaissance avec ce riche répertoire.

Pourquoi ces trois pièces ? Chacunereprésente un genre dans le théâtre préshakespearien : The Longer porte la marque des pièces morales (ou moralités, ou encore «moral interludes»), Mother Bombie est une pièce de cour, et Orlando une sorte de chronique légendaire ; chacune se définit dans une tradition spectaculaire, mais un aspect les relie : la folie en est le thème ou le personnage central.

L'analyse du concept de folie dans ces trois pièces se révèle d'autant plus enrichissant que sa polysémie s'y exprime clairement. Dans un article remarquablement documenté sur cette polysémie, Louis Roux cite Blackstone

The idiot is one that hath had no understanding from his nativity : the lunatic is one who hath had understanding, but... hath lost the use of his reason. ${ }^{1}$.

A ces deux sens, qui éclairent la notion de folie dans Mother Bombie pour le premier, et Orlando pour le second, il faut ajouter un troisième pour comprendre le person- 
nage central de The Longer, personnage au nom révélateur: Moros. Présenté comme un sot, né sot, iln'est pas cependant le simple d'esprit figuré par les deux sots de Mother Bombie. Moros s'identifie aux vierges folles du Nouveau Testament, persistant dans la folie, il est «past redemption», alors que Orlando, déraisonnable dans sa jalousie, perd à juste titre sa raison, mais sort victorieux de l'épreuve. Les trois pièces poursuivent un but didactique évident, la folie des personnages désigne l'erreur aux autres personnages, et aux spectateurs et les renseigne sur leur propre folie. Ainsi, dans chaque pièce, bien que l'accent soit mis sur un sens particulier du terme, celui-ci conserve sa polysémie et l'on garde toujours présent à l'esprit les tableaux des différentes versions de La Nef des Fous, ou encore de L'Eloge de la folie d'Erasme ${ }^{2}$. Nous montrerons au passage l'emploi judicieux de la musique dans trois pièces de tradition et d'auteurs différents. Dans un théâtre qui offre des aspects si disparates, la présence d'un facteur d'unité est toujours rassurante.

Peut-être est-il sage, en abordant chaque pièce d'en donner un bref résumé. The Longer Thou Livest, The More Fool Thou Art date de 1559 ; elle est attribuée à W. Wager ${ }^{3}$.

Disciple, à l'aide de Piété et Exercice de la Vertu («Exercitation»), tente la lourde tâche de réformer Moros, jeune sot qui ne voit aucune bonne raison à l'étude des Ecritures. Discipline se rend compte que son attitude vient en partie de son absence d'éducation, et blâme surtout ses parents. Moros ne veut clairement rien entendre, préférant une vie de chansons, danses et loisirs. Discipline n'hésite pas alors à faire appel à la violence. Moros joue le jeu et accepte le catéchisme que lui donne Discipline. Les vertus ne semblent pas très optimistes, et dès qu'elles ont le dos tourné, Moros recherche une compagnie plus divertissante. Il la trouve en la personne de Oisiveté qui, avec ses compères Colère et Incontinence, lui offre sa protection contre les coups de Discipline que Moros défie dès qu'il le rencontre à nouveau. Moros passe ainsi à l'âge adulte, et Fortune, qui ne regarde pas au mérite, décide de le combler de ses faveurs. Les trois vices du début sont alors remplacés par trois vices de l'âge adulte de Moros : Ignorance, Impiété, et Cruauté. A leur contact, la jeune insouciance de Moros se transforme en vanité et stupidité. En tant qu'homme, et gentilhomme de surcroît, sa folie est d'autant plus condamnable qu'elle oppresse le peuple. Un nouvel affrontement avec Discipline le montre, malgré son impuissance à vaincre Discipline, résolu à ne pas changer le cours de sa vie. Jusque dans son troisième âge il cherche à prouver qu'il est un homme et ne se bat plus que contre lui-même : son insouciance, son énergie de jeune sot n'est plus que violence insensée. Il est temps, à présent, d'envoyer le châtiment : Jugement de Dieu prononce la sentence. Moros se sent mal, mais ne comprend toujours pas. Une dernière chance lui reste de se repentir, mais dans sa folle ignorance, il ne sait que demander une coupe de vin. Confusion lui passe l'habit des fous ; acceptant son sort, il réclame lui-même le Diable, vers qui il s'en va, perché sur le dos de Confusion. Les vertus concluent alors la pièce.

Dans une pièce où le protagoniste s'appelle Moros, il n'est pas étonnant de 
trouver les termes «fool», «foolish», «folly», à toutes les pages ou presque. Il faut cependant savoir ce que ces termes recouvrent. A observer le vocabulaire de la pièce, on voit vite que ces termes ne se posent pas en équivalents de «madness» (mot qui n'apparaît qu'une fois) et ne s'opposent pas à «sanity», mais plutôt à «wisdom» (7 occurrences), «sapience» (14 occurrences), «wise» (17 occurrences), «wisement» (21 occurrences). Il s'agit bien de l'opposition «foolish»/«wise», telle qu'elle $s$ 'exprime dans la parabole des vierges folles et des vierges sages (Evangile selon St Matthieu, ch. 25). En vérité, la tâche de Discipline est de préparer Moros à la venue du Fils de l'Homme car, selon St Matthieu :

Ye know neither the day nor the hourwherein the Son of Man cometh

Ce qui frappe chez Moros est son absence de grâce, qui lui manque depuis le début. L'auteur le déclare dès le prologue, et le rappelle au cours de la pièce : Dieu seul décide, par sa grâce, de sauver une âme, encore faut-il que cette âme ait été prédisposée à recevoir cette grâce. Cette prédisposition manque à Moros :

But nothing, God except is so strong as nature ;

For neither counsel, learning nor sapience

Can an evil nature to honest manners allure

(Prologue, II. 44-46)4.

Confusion condamne Moros en ces termes :

Thy malice will not let thee thy folly to see, So that thou hast not the grace thy life to amend.

(II. 1837-1838)

Dans son introduction de la pièce, Mark Benbow souligne que l'affrontement Vertus/Vices pour l'âme de Moros ne représente qu'un choix illusoire pour lui (p. XV). Pourtant, Wager prolonge cette illusion en présentant un personnage ambigu quant à la nature de sa folie. Lorsqu'il entre en scène, chantant des bribes de ballades ( $\ll$ the foot of many songs as fools were wont $»$ ) il se présente à nous comme un sot, un simple d'esprit, les divagations musicales étant un signe de folie ou de simplicité d'esprit (Shakespeare, on le sait, s'en sert pour le personnage d'Ophélie, dans Hamlet, IV, V). Pourtant, par certaines de ses remarques, Moros ne nous semble pas dépourvu d'esprit ; on peut mettre sur le compte de sa jeunesse et de son innocence certains de ses bons mots, et penser qu'il ne se moque pas, en fait, des vertus : la tradition de L'Eloge de la folie veut que la vérité sorte de la bouche des 
sots. Mais sa dissimulation montre qu'il se soucie plus de sauver sa peau que son âme. Du reste, les vertus sont d'accord : si Moros accepte de suivre leur enseignement, elles peuvent le sauver:

\section{And though $\times$ Moros» a fool doth signify \\ Yet you may be wise, as I trust you wille.}

(II. 271-272)

La véritable folie de Moros consiste à refuser de se laisser gagner par la grâce. D'une folie toute de jeunesse, due surtout à un manque d'éducation (le rôle des parents dans la chute de Moros est discuté à de nombreuses reprises), et ne suivant après tout que les vices de la jeunesse, Moros passe à une folie plus consciente en suivant les vices de l'âge adulte. Dans le schéma traditionnel des pièces morales, le protagoniste, ayant commencé de son plein gré son éducation vertueuse, se trouve confronté aux vices dont il adopte les préceptes. Leur accord, scellé par une chanson à laquelle le protagoniste participe, marque sa chute ; plus tard, à nouveau sous l'emprise des vertus, il se repent et les vices sont châtiés. La disposition même du protagoniste pour la vertu et le bien, fait apparaître sa chute comme une faiblesse passagère. Pour Moros, il n'en estrien : sa première conversion est feinte, sa chute est double. Discipline continue de le considérer comme «a natural fool», même après sa première chute qui marque son évolution vers l'âge adulte. Moros se sent bien fragile encore, et ne parvient que difficilement à faire face à Discipline, mais son choix est fait, et sa folie s'installe dans de nouveaux vices bien plus graves : sa folie devient elle-même un vice, celui-là même qui le condamne. A chaque tournant de la pièce, les vertus reprennent l'adage : «The longer thou livest, the more fool thou art». La seconde chute de Moros, également marquée par une chanson à plusieurs voix, dénote cette folie comme la persévérance dans l'erreur, le refus de la grâce. Discipline ne peut plus que prédire sa damnation. Le jeu de Moros se transforme, et sa folie s'extériorise, elle est représentée comme «madness». Au tout dernier moment, alors qu'il est trop tard, Moros, revêtu de l'habit de fou, se retrouve à la case départ, comme si sa vie n'avait été qu'un songe :

Where is my goodly gear?

I see well that I was asleep indeed.

What, am I fain a fool's coat to wear?

We must learn at «Christ's cross me speed*

Other I was a gentleman and had servants

Or else I dreamed that I was a gentleman.

(II, 1839-1844).

La référence à «Christ's cross me speed» renvoie au catéchisme que 
Discipline lui avait donné à apprendre au début de la pièce.

Après le départ de Moros pour l'enfer, Exercice de la Vertu et Piété rapprochent le sot, le fou qui refuse la sagesse, de l'aliéné mental, le «madman» :

Exercitation : A wicked man having learning and cunning

And doth many sciences understand

Is like one whose wits are running,

I mean a mad man having a sword in his hand.

Piety : For as a mad man in his hand edge-tool

Seeketh both himself and other to kill,

So a cunning man without wisdom is but a fool,

For both himself and many other he doth spill.

(II. 1943-1950)

Pour Moros donc, tout simple d'esprit, «innocent», qu'il puisse paraître au début de la pièce, il n'est point de salut, car sa folie l'oppose à toute raison, toute sagesse, et l'enferme dans un rejet de la grâce divine. Alors, il devient comparable au fou, à l'aliéné, qui ne sait plus ce qu'il fait, détruit tout autour de lui, et se détruit lui-même.

D'une toute autre veine, la comédie de John Lyly, Mother Bombie, déplace le problème sur le plan social.

Memphio cherche à marier son fils Accius, simple d'esprit («a fool»), et pense à la fille de Stellio, Silena, si belle, que son père la garde enfermée dans la maison. Il ne sait pas que la véritable raison de cet enfermement est la sottise naturelle de Silena. Stellio, de son côté, voit ce mariage d'un bon œil, ne sachant pas que Accius est également un sot. Une autre paire d'amants entre en jeu : Candius et Livia. Ils s'aiment, mais n'ont pas le consentemnet de leur pères respectifs qui, eux, veulent qu'ils épousent l'un Silena , l'autre Accius. Les serviteurs, au courant de toute l'affaire, décident de prendre partie pour Candius et Livia, et d'encourager le mariage d'Accius avec Silena. Le problème de l'identité, qui apporte la solution finale, est soulevé par Silena qui, dans son innocence, se pose des questions : elle va voir Mother Bombie, sorte d'oracle, pour savoir si elle est fille. A mots couverts, comme le font les oracles, Mother Bombie lui dit une vérité que Silena ne peut ni comprendre, ni reconnaître. Une troisième paire d'amoureux entre alors en jeu : Maestius et Serena : étant frère et sœur, leur amour est condamné. Ils se rendent chez Mother Bombie qui leur prédit leur mariage prochain. Après des échanges de costumes et d'identités, la vérité éclate : Vicinia, mère supposée de Maestius et Serena, et nourrice des enfants de Memphio et Stellio, avait fait l'échange avec ses propres enfants, Accius et Silena ; les deux sots sont donc frère et sœur : leur mariage est impossible, mais rend possible l'union de Maestius et Serena, et celle de Candius 
et Livia. Memphio et Stellio promettent de continuer à s'occuper des deux sots.

Le terme le plus souvent employé dans la pièce est «fool». D’autres termes, beaucoup moins fréquents, définissent cette «folie» de Accius et Silena, la confinant dans le domaine de la simplicité d'esprit : «simple», «simplicitie», «idiots». Les termes «mad», «lunatike», «lunasie» n'apparaissent que très peu. Innocents dans tous les sens du mot, les deux sots sont victimes des manipulations des prétendus «sages»: les termes «wise», «wisely», s'opposent ici également à «fool» et ses dérivés. Le texte met en jeu la polysémie du terme «fool», car, sans nier la simplicité d'esprit de Accius et Silena il montre les autres personnages enfermés dans leur propre folie qui, elle, n'a plus l'excuse de la simplicité naturelle des deux sots. Les parents, par leur cupidité, leur entêtement, secouvrent de ridicule, et agissent comme des sots; en donnant le dernier mot à Silena, l'auteur excuse les deux sots, et condamne les parents :

Accius : Come, sister, the best is, we shall have good chere these foure dayes.

Lucio : And be fooles for ever.

Silena : Thats none of our upseekings.

(Bond, V, iii, 399-402)

Grâce à leur savoir, leurs connaissances, mis en commun, les serviteurs possèdent, sinon la sagesse, du moins suffisamment d'esprit pour savoir ce qu'ils doivent faire. Avec grand plaisir, comme à l'accoutumé, ils prennent parti contre leurs maîtres :

Dromio : I lacke but a wagge more to make of my counsell, and then you shall see an exquisite Coosnage, and the father more fool than the sonne.

(Bond, I, i, 93-95)

Plus tard, parlant de Risio, autre serviteur :

His knaverie and my wit, should make our masters that are fooles beggers, and us two that are bond, free.

(Bond, II, i, 5-7)

La «folie» des maîtres tient de cette vanité de se croire plus sage que les autres. En cela, ils s'approchent de la folie de Silena, qui est la mieux étudiée dans la pièce. Lorsque Stellio s'ouvre de ses plans (on pourrait dire de ses machinations) à l'égard de sa fille, à son valet Risio, ils discutent longuement de cette folie. Risio 
ne cache pas l'opinion qu'il a de son maître, et donne un double sens au dialogue :

Stellio : Doest thou thinke she tooke her foolishness of me? Risio : $I$, and so cunningly, that she toke it not from you.

(Bond, I, ii)

Risio pense que Silena est sotte de naissance, et qu'il ne se trouve aucun remède à sa folie. Stellio tente de minimiser la chose, et ne voit chez elle que le résultat de sa vanité : elle se croit subtile et sage, telle est sa folie : se faisant, il décrit sa propre folie, et celle des autres pères de la pièce.

Lorsque Candius, ayant promis de courtiser Silena, la rencontre pour la première fois, il a du mal à analyser son malaise face à elle. Tout d'abord il la prend pour une excentrique, puis pour une folle, enfin, il se rend compte de la véritable nature de sa folie :

Now I perceive thy folly, who hath rakt together all the odd blind phrases, that helpe them that knowe not how to discourse but when they cannot answer wisely, eyther with gybling cover their rudenesse, or by some new coyned by-word bewraie theyr peevishnesse.

(Bond, II, iii, 68-72)

Avec ses «odd blind phrases», Silena rejoint Moros et ses «foot of many songs». Le discours de Silena peut paraître insensé, sans queue ni tête, puisqu'elle ne sait pas où son corps se situe :

For Ihave asfayre aface as ever trode on shoe sole, and asfree afoote as ever lookt with two eyes.

(Bond, II, iii, 19-20)

Mais ceci n'est qu'un aspect de sa recherche de sa propre identité. Silena tente désespérément de se définir, désirant sans cesse se donner une identité, une volonté, se prouver à elle-même qu'elle est bien Silena, fille de Stellio : sans le savoir, elle touche au fond du problème.

Cette préoccupation pour la définition de l'identité est au cœur de nombreuses pièces de John Lyly. On sait que l'authenticité des chansons publiées par Blount dans l'édition de six comédies de John Lyly en 1632, a été contestée : la polémique suit son cours. Pourtant, l'échange musical entre Silena et Accius paraît tout à fait caractéristique : Silena parle d'identité et d'apparence, d'identité exprimée dans des attributs : 
O Cupid ! Monarchover Kings wherefore hast thou feet and wings?

It is to shew how swift thou art

When thou woundest a tender heart:

Thy wings being clip't, and feete held still,

Thy Bow so many could not kill.

Cupidon ne serait plus Cupidon, bien que toujours l'Amour. Or, Silena n'est pas Silena, et confusément elle le sent bien, mais le changement d'identité ne lui rend pas la sagesse, elle reste simple d'esprit. Quantà Accius il se dévoile comme sot, de façon subtile, justifiant sa «folie» par l'Amour, pouvant faire croire à ceux qui assistent de loin à l'entrevue qu'il est tout à fait sain d'esprit :

It is all one in Venus wanton Schoole, Who highest sits, the wise man or the foole :

Fooles in loves Colledge

Have farre more knowledge,

To reade a woman over,

Than a neate prating lover.

Nay tis confest,

That fooles please women best.

(Bond, III, iii, 1-14)

Mother Bombie apparaît comme dépositaire de la sagesse, de la vérité, connaissant bien la folie du monde. Tous les personnages qui vont la voir, persuadés qu'elle détient la vérité, sont pourtant si prisonniers de leur folie, qu'ils deviennent incapables de reconnaître cette vérité. Même Vicinia, tourmentée par sa conscience, prévenue par Mother Bombie, ne se décide qu'au dernier moment, à confesser sa folie, pour prévenir l'inceste qui résulterait du mariage de Accius et Silena. L'ordre rétabli, les deux sots se contentent de leur sort, ils resteront sots toute leur vie, mais les autres ont-ils vraiment trouvé la sagesse?

Orlando Furioso est une adaptation tout à fait libre d'un épisode du Roland Furieux de l'Arioste.

Marsilius ayant rassemblé tous les prétendants de sa fille, Angélica, il accorde sa main à celui qu'elle choisira. Orlando parle le dernier, et bien que ni roi, ni empereur, il l'emporte sur les autres prétendants. Aussitôt les rois se retournent contre Orlando et Marsilius, à qui ils déclarent la guerre. Sacrapant, homme vain qui se croit au-dessus des rois, égale des dieux, imagine un stratagème pour se débarrasser d'Orlando, et obtenir Angélica et la couronne. Il rend Orlando jaloux en accrochant aux arbres des ballades et billets doux laissant croire à une liaison amoureuse entre Médor et Angélica. Orlando plonge dans la folie en découvrant ce 
qu'il croit être l'infidélité de sa dame.

Pendant sa folie, la situation ne s'arrange pas pour Marsilius qui n'a plus, pour le soutenir, que Mandricard. Angélica, bannie, erre dans la forêt. Telle est la situation lorsque les Pairs de France rencontrent Marsilius et Mandricard, déguisés en pèlerins. Il n'hésitent pas à le suivre lorsque Marsilius révèle son identité : il est temps de retrouver Orlando, et de le venger. Mais seul le héros peut accomplir dignement cette vengeance, rétablir Marsilius et innocenter Angélica. Pour cela, il lui faut retrouver la raison. Mélissa descend des cieux, l'endort, et, à l'aide de musique et de danse, lui envoie une vision et le fait revenir à lui. Apprenant la trahison de Sacrapant, il le provoque et le tue. Avant de mourir, le traître disculpe Angélica, Orlando, déguisé en berger, se lance à sa recherche. Il la trouve aux mains des Pairs, tout disposés à la mettre à mort. Orlando les défie, et, devant sa force et son courage, il paraît clair à tous qu'il est Orlando. Il se révèle à eux et leur explique toute l'intrigue.

Deux folies s'opposent : celle de Sacrapant et des rois, refusant le choix d'Angélica, et se rebellant contre un roi légitime. Cette folie les pousse à des actes extrêmes et les conduità leur perte. L'honneur mal placé et la richesse ont transformé ces hommes en monstres d'orgueil. Ils perdent tout sens de jugement. Ainsi, Rodomant ne peut concevoir qu'Orlando, simple chevalier errant, puisse le vaincre. Dans leur folie, ils confondent valeur avec richesse et rang. La leçon paraît claire lorsqu'à la fin, les Pairs de France reconnaissent la valeur en Marsillius et Mandricard, malgré leur déguisement de pèlerins :

Though habit be but meane, their mindes excell.

(II. 1038)

Tout comme Moros, les rois sont condamnés dès le début, à moins que, touchés par la grâce, ils ne se repentent. Un seul accepte de s'ouvrir à la grâce, Mandricard, touché par la figure rédemptrice de Marsilius.

Face à cette folie destructrice, celle d'Orlando nous apparaît comme une épreuve purificatrice. Il s'agit d'une folie provoquée par la passion : la jalousie. Orlando, homme sain d'esprit jusque-là, perd la raison : ici «folie» signifie démence. La description de la folie d'Orlando a été critiquée en ces termes :

In delineating the madness of Orlando, Greene is wholly untrue to nature and shows no knowledge at all of the psychology of insanity. (Churton Collins, Introduction aux Euvres complètes).

Certes, le comportement d'Orlando ne convaincrait aucun psychiatre, et il ne s'agit pas pour Greene de faire une analyse pathologique dont il était vraisemblablement 
incapable, et tous les spectateurs avec lui. Greene fait mieux que cela : il accumule sur son personnage tout ce qui, dans l'esprit populaire, se rattache à la démence. Orlando assume les attributs de la folie, comme Moros revêt le costume du fou. Extravagance, humeur changeante, délire, logique différente qui échappe à son entourage, et qui suit les cheminements de sa passion et de son tourment, idée fixe, lycanthropie même, ${ }^{7}$ et comportement violent se succèdent. Lorsque Orgalio apprend à Marsilius qu'Orlando a perdu l'esprit, il ne peut que le comparer à un autre «fou» légendaire :

He, my lord, runs madding through the woods

Like mad Orestes in his greatest rage.

Il serait facile de faire d'Orlando un pantin ridicule, dont on peut aisément se moquer, comme croient pouvoir le faire les deux clowns qui le traitent comme l'idiot du village. Pourant, par cette accumulation des traits de la folie, par l'ampleur même que donne cette accumulation à sa démence, Orlando conserve sa dignité et son autorité. Il reste le chevalier errant (cette fois à plus d'un titre) et, avec son armée de fortune (les deux clowns rangés sous son autorité), met en fuite Rodomant et Brandimart, alors qu'ils s'apprêtaient à faire un mauvais sort à Angélica. Orgalio se sent démuni face à cette folie. Il tente à plusieurs reprises de ramener Orlando à la raison, ou de devancer ses désirs, mais il raisonne en sage, et ne voit pas que la logique du fou n'a rien à voir avec la sienne; Il veut prévenir l'instabilité qu'il sent bien être au cœur de la folie d'Orlando, mais ne perçoit pas que cette instabilité même possède une logique interne : lorsque Orlando sauve Angélica, déguisée en vieille femme, mais reconnue par Rodomant et Brandimart, lui ne la reconnaît pas, pourtant il la cherche, elle est son idée fixe de vengeance. Lorsque Orgalio lui présente un des clowns habillé en femme, il le croit sur parole puisqu'il lui dit qu'il s'agit d'Angélica, et rosse le clown. Même fou, Orlando reste le chevalier : dans son désir de vengeance, il ne peut lever la main sur une femme ; le clown lui permet d'exercer sa folle violence.

La façon dont Orlando est guéri révèle sa folie comme une épreuve. La scène se passe en deux temps. Orgalio, toujours plein d'espoir, fait venir un musicien pour tenter d'apaiser Orlando, sinon lui rendre la raison. On connaît les pouvoirs thérapeutiques que l'on accorde à la musique, pourtant, Orlando, loin d'être apaisé, se rue sur le ménestrel et lui casse son violon sur la tête. Mais, lorsque Mélissa lui envoie sa musique, il trouve d'abord le repos, puis la raison. L'explication passe par le système des trois musiques : musica mundana, musica humana, musica instrumentalis. La musique du monde, ou harmonie céleste, parfaite, n'est pas audible par le commun des mortels ; la musique humaine représente l'équilibre du corps et de ses composants, ainsi que de l'esprit qui, dans le cas d'Orlando, est dérangé ; la musique des instruments (et la voix en fait partie) est en fait la musique que les 
mortels pratiquent, dans le vain espoir de retrouver l'harmonie céleste. La croyance que certaines échelles mélodiques provoquaient chez l'homme un déséquilibre, ou un équilibre des passions, remonte à l'antiquité, et c'est de cette croyance que la musique détient son pouvoir thérapeutique. Le ménestrel, envoyé en toute bonne foi par Orgalio, se présente au moment où Orlando n'a pas épuisé toute sa passion et sa rage. Lorsque Mélissa entre en jeu, elle le purifie, et sa musique n'est plus la musica instrumentalis, mais la musique céleste qu'Orlando peut entendre à présent. Mélissa, l'abeille, est, selon Virgile, une émanation de l'âme divine :

On a dit que les abeilles avaient en elles une parcelle de l' âme divine et des émanations de l'empyrée.

(Les Géorgiques, livre IV) ${ }^{8}$

et chez le néoplatonicien Porphyre, le nom de Mélissa est attaché à l'âme pure des initiés. Mélissa se trouve ici élevée au rang de déesse, et Orlando la reconnaît comme telle :

Thanks sacred Goddes for thy helping hand.

(II. 1217)

Cette apparition de la déesse qui vient effacer la folie de l'esprit d'Orlando, et ainsi rétablir l'ordre et la vérité, peut paraître mécanique. Il s'agit d'une représentation spectaculaire de la Providence, d'une transposition du personnage envoyé par Dieu dans les pièces morales, pour récompenser ou punir le protagoniste et rétablir 1'ordre : Jugement de Dieu (The Longer), Récompense (Apius and Virginia), Sévérité (Like Will to Like), Miséricorde (Mankind), représentent, comme Mélissa et Mother Bombie, l'intervention de la Providence divine. La folie des personnages les conduit dans une impasse : seule la grâce de Dieu peut les en sortir ; celui qui la rejette se damne, celui qui se laisse pénétrer par elle est sauvé.

Qu'apprennent ces personnages désignés comme fous, dans leur folie ? Moros n'apprend rien, sinon à accepter sa damnation, mais on peut penser qu'il s'y précipite dans une folle ignorance de ce qui l'attend. Accius et Silena apprennent la résignation jusqu'au contentement. Orlando atteint les sommets de la grâce. Plus important peut-être, leur folie fait éclater aux yeux de tous la Vérité. Car la folie est aussi une possession par le mensonge; le fou peut montrer ou dire la vérité que chacun cache, puisqu'on peut le taxer de menteur. Première étape vers la Vérité, la folie expose le mensonge et constitue une épreuve à laquelle les personnages doivent se soumettre pour accéder à cette Vérité suprême. Dans un ordre théologique et providentiel, la folie trouve sa place, son sens. 


\section{NOTES}

1 Voir Louis Roux, «Divagations sémantiques pour un siècle de raisons», in Folie, Folies, Folly dans le monde anglosaxon américain aux XVIIe et XVIIIe siècles, Actes du colloque de la société d'études anglo-américaines des XVIIe et XVIIIe siècles, 22 et 23 octobre 1982 (Aix-en-Provence : Université de Provence, 1984), p. 8.

2 Au sujet de La Nef des Fous, on pourra consulter l'ouvrage de Fr. Aurelius Pompen, The English Versions of the Ship of Fools (New York : Octagon Books, 1967 ; a l'origine publié par Longman, Green and Co, 1925).

3 Pour les attributions et les dates nous suivons les données de Alfred Harbage, Annals of English Drama, 975-1700, rev. par S. Schoenbaum (Londres : Methuen, 1964).

4 Les références sont à l'édition de R. MarkBenbow, Regents Renaissance DramaSeries (Londres : Arnold, 1968).

5 Les références sotn à l'édition de R. Warwick Bond, The Complete Works of John Lyly, 3 vol. (Oxford : Clarendon Press. 1902).

6 Les références sont à l'édition de J. Churton Collins, The Plays and Poems of Robert Greene, 2 vol. (Oxford : Clarendon Press, 1905).

7 Jean Fuzier fait remarquer qu'il s'agit là d'un des trois cas de lycanthropie dans le thêâtre élisabéthain et jacobéen.

8 Virgile, Les Géorgiques, trad. E. de Saint-Denis (Paris Les Belles Lettres, 1963). 